УДК 517.53

K. G. Malyutin, A. A. Revenko

\title{
EXTREMAL PROBLEMS IN THE SPACE OF MEROMORPHIC FUNCTIONS OF FINITE ORDER IN THE HALF-PLANE
}

\author{
Dedicated to the 90th anniversary of Anatoly A. Gol'dberg
}

\begin{abstract}
K. G. Malyutin, A. A. Revenko. Extreme problems in the space of meromorphic functions of finite order in the half-plane, Mat. Stud. 52 (2019), 144-155.

The extremal problems in the space of meromorphic functions of order $\rho>1$ in upper half-plane are studed. The method for studying is based on the theory of Fourier coefficients of meromorphic functions. The concept of just meromorphic function of order $\rho>1$ in upper half-plane is introduced. Using lemma on the Pólya peaks and the Parseval equality, sharp estimate from below of the upper limits of relations Nevanlinna characteristics of meromorphic functions in the upper half plane are obtained. An inequality related to an analogue of the Nevanlinna problem for half-plane is proved.
\end{abstract}

1. Introduction. In this paper, we use the Fourier series method for the study of extremal problems in the space of meromorphic functions of finite order $\rho>1$ in the upper half-plane $\mathbb{C}_{+}=\{z: \operatorname{Im} z>0\}$. This method was introduced by L. A. Rubel and B. A. Taylor [1]. Further the Fourier series method was used by J. B. Miles, D. P. Shea, A. A. Kondratyuk [2], [3], [4], Ya. V. Vasyl'kiv and others. At the beginning of the 20th century, the first author of this paper extended the results of L. A. Rubel, B. A. Taylor, J.B. Miles to delta-subharmonic functions in the half-plane $\mathbb{C}_{+}[5]$. In 1973, J. B. Miles and D. P. Shea [6] considered an extremal problem in value distribution theory of meromorphic functions. Let $f$ be a meromorphic function in complex plane and let

$$
N(r, f)=\sum_{\left|b_{k}\right| \leq r} \log \frac{r}{\left|b_{k}\right|}
$$

be the Nevanlinna counting function of poles $\left(b_{k}\right)$ of the function $f$,

$$
\begin{gathered}
m(r, f)=\frac{1}{2 \pi} \int_{0}^{2 \pi} \log ^{+}\left|f\left(r e^{i \theta}\right)\right| d \theta, \text { where } a^{+}= \begin{cases}a^{+}=a, & a>0, \\
a^{+}=0, & a \leq 0,\end{cases} \\
T(r, f)=m(r, f)+N(r, f), \quad m_{2}(r, f)=\left\{\frac{1}{2 \pi} \int_{0}^{2 \pi}|\log | f\left(r e^{i \theta}\right)||^{2} d \theta\right\}^{1 / 2} .
\end{gathered}
$$

2020 Mathematics Subject Classification: 30D15, 30D30, 30D35.

Keywords: extremal problem; meromorphic function of finite order; complete measure; Pólya lemma; Carleman formula; Nevanlinna characteristic; Parseval equality.

doi:10.30970/ms.52.2.144-155

(C) K. G. Malyutin, A. A. Revenko, 2019 
Theorem MSh (Miles and Shea [6]). Let $f$ be a meromorphic function in complex plane of order $\rho, 0<\rho<\infty$. Then

$$
\limsup _{r \rightarrow \infty} \frac{N(r, f)+N(r, 1 / f)}{m_{2}(r, f)} \geq \frac{|\sin \pi \rho|}{\pi \rho} \sqrt{2 /\left(1+\frac{\sin 2 \pi \rho}{2 \pi \rho}\right)},
$$

and this inequality is exact, i.e. for some meromorphic function $f$ of order $\rho$ equality holds.

We also give a corollary of Theorem MSh related to one Nevanlinna problem, which has not yet been solved in the general case and consists in finding the exact lower bound for the quantity

$$
\varkappa(f)=\limsup _{r \rightarrow \infty} \frac{N(r, f)+N(r, 1 / f)}{T(r, f)} .
$$

If $f$ is an entire function then $\delta(0, f)=1-\varkappa(f)$, where $\delta(0, f)$ is the Nevanlinna deficiency at zero, i.e.

$$
\delta(0, f)=\liminf _{r \rightarrow \infty} \frac{m(r, 1 / f)}{T(r, f)} .
$$

The estimate of $\varkappa(f)$ given below is the best known [7, p. 63].

Corollary MSh (Miles and Shea). Let $f$ be a meromorphic function in the complex plane of order $\rho, 1<\rho<\infty$. Then

$$
\varkappa(f) \geq 0.9 \frac{|\sin \pi \rho|}{1+\rho} .
$$

We prove a similar statements for functions defined in the upper half-plane. We denote by $C(a, r)$ the open disk of radius $r$ with the center at $a$, and by $\Omega_{+}$the intersection of a set $\Omega$ with the half-plane $\mathbb{C}_{+}: \Omega_{+}=\Omega \cap \mathbb{C}_{+}$.

A function $f$ is called just analytic function in $\mathbb{C}_{+}$if $f$ is analytic function in $\mathbb{C}_{+}$and $\limsup \log |f(z)| \leq 0$ for any $t \in(-\infty, \infty)$. By $J A$ we denote the space of just analytic $z \rightarrow t, z \in \mathbb{C}_{+}$ functions in $\mathbb{C}_{+}$.

Let $A K[8]$ be the space of analytic functions in $\mathbb{C}_{+}$such that $\log |f(z)|$ has a positive harmonic majorant in any bounded domain in $\mathbb{C}_{+}$. The functions $f$ of the space $A K$ have the following properties:

a) $\log |f(z)|$ has non-tangential limit $\log |f(t)|$ almost everywhere on the real axis such that $\log |f(t)| \in L_{l o c}^{1}(-\infty, \infty)$;

b) for any $f \in A K$ there exists

$$
\lim _{y \rightarrow+0} \int_{a}^{b} \log |f(t+i y)| d t=\nu([a, b])
$$

where $\nu$ is a signed measure on the real axis, $a, b \in(-\infty,+\infty) \backslash E_{f}, E_{f}$ is a countable set. The measure $\nu$ is called the boundary measure of the function $f$;

c) $d \nu(t)=\log |f(t)| d t+d \sigma(t)$, where the measure $\sigma$ is singular with respect to the Lebesgue measure. 
For the function $f \in A K$, following [8], we define the complete measure $\lambda$ of $f$ as

$$
\lambda(K):=\lambda_{f}(K)=2 \pi \int_{\mathbb{C}_{+} \cap K} \operatorname{Im} \zeta d \mu_{f}(\zeta)-\nu(K),
$$

where $\mu_{f}$ is the Riesz measure of the function $\log |f(z)|$. The measure $\lambda$ has the following properties:

1) $\lambda$ is a finite measure on each compact subset $K$ of $\mathbb{C}$;

2) $\lambda$ is a positive measure outside $\mathbb{R}$;

3) $\lambda$ is a zero measure on the half-plane $\mathbb{C}_{-}=\{z: \operatorname{Im} z<0\}$.

Conversely, if a measure $\lambda$ with properties 1)-3) is given, then there exists a function $f \in A K$ such that $\lambda$ is the complete measure of $f$.

The space $J M$ of just meromorphic functions in $\mathbb{C}_{+}$is defined as $J A / J A$. It is true [8]: $J A \subset A K$ and $J M=A K / A K$.

For a fixed measure $\lambda$ let

$$
d \lambda_{m}(\zeta)=\frac{\sin m \varphi}{\sin \varphi} \tau^{m-1} d \lambda(\zeta)\left(\zeta=\tau e^{i \varphi}\right), \lambda_{m}(r)=\lambda_{m}(\overline{C(0, r)}),
$$

where $\frac{\sin m \varphi}{\sin \varphi}$ is defined for $\varphi=0, \pi$ by continuity.

Let $f \in J M$ and let $\lambda=\lambda_{f}$ be the corresponding complete measure of $f$. The next relation is the Carleman's formula in Grishin's notations [8]:

$$
\frac{1}{r^{k}} \int_{0}^{\pi} \log \left|f\left(r e^{i \varphi}\right)\right| \sin k \varphi d \varphi=\int_{r_{0}}^{r} \frac{\lambda_{k}(t)}{t^{2 k+1}} d t+\frac{1}{r_{0}^{k}} \int_{0}^{\pi} \log \left|f\left(r_{0} e^{i \varphi}\right)\right| \sin k \varphi d \varphi, \quad k \in \mathbb{N}
$$

where $r_{0}>0$ is an arbitrary (usually fixed) positive number (we can set $r_{0}=1$ ).

In particular, for $k=1$ we have

$$
\frac{1}{r} \int_{0}^{\pi} \log \left|f\left(r e^{i \varphi}\right)\right| \sin \varphi d \varphi=\int_{r_{0}}^{r} \frac{\lambda(t)}{t^{3}} d t+\frac{1}{r_{0}} \int_{0}^{\pi} \log \left|f\left(r_{0} e^{i \varphi}\right)\right| \sin \varphi d \varphi
$$

for all $r>r_{0}$.

For $f \in J M$, let $\lambda_{f}:=\lambda=\lambda_{+}-\lambda_{-}$be the Jordan decomposition of $\lambda_{f}$. We set up following notations and terminology

$$
\begin{gathered}
m(r, f):=\frac{1}{r} \int_{0}^{\pi} \log ^{+}\left|f\left(r e^{i \varphi}\right)\right| \sin \varphi d \varphi, \quad N\left(r, f, r_{0}\right):=\int_{r_{0}}^{r} \frac{\lambda_{-}(t)}{t^{3}} d t, \\
T\left(r, f, r_{0}\right):=m(r, f)+N\left(r, f, r_{0}\right)+m\left(r_{0}, \frac{1}{f}\right), \quad r>r_{0},
\end{gathered}
$$

where $r_{0}$ is an arbitrary fixed positive number, which we shall drop in the notations (provided that this cannot lead to confusion; for instance, we shall write $T(r, f)$ in place of $T\left(r, f, r_{0}\right)$ and so on). 
In this notation the Carleman's formula (2) can be written as follows:

$$
T(r, f)=T\left(r, \frac{1}{f}\right) .
$$

Definition 1. A function $f \in J M$ is said to be a function of finite order if there exists a positive constant $\beta>1$ such that the inequality $T(r, f) \leq r^{\beta-1}$ is valid for all sufficiently large values of $r$ (i.e $r>r_{0}(\beta)$ ).

The greatest lower bound $\rho$ of such numbers $\beta$ is called the order of the function $f \in J M$. It is clear that $\rho \geq 1$.

It follows from this definition that if $\rho$ is the order of the function $f \in J M$, and if $\varepsilon$ is an arbitrary positive number, then

$$
r^{\rho-1-\varepsilon}<T(r, f)<r^{\rho-1+\varepsilon},
$$

where the inequality on the right is satisfied for all sufficiently large values of $r$, and the inequality on the left holds for some sequence $\left\{r_{n}\right\}$ of values of $r$, tending to infinity. It is easy to verify that condition (4) is equivalent to the equation

$$
\rho=\limsup _{r \rightarrow \infty} \frac{\log T(r, f)}{\log r}+1 .
$$

The space of such functions is denoted by $J M(\rho)$. By $J A(\rho) \subset J M(\rho)$ we denote the subspace of just analytic functions in $\mathbb{C}_{+}$.

It follows from (3) and the definition of the space $J M(\rho)$ : if $\rho$ is the order of the function $f \in J M$, and if $\varepsilon$ is an arbitrary positive number, then for all sufficiently large values of $r>r_{0}(\varepsilon)$

$$
m(r, f)<r^{\rho-1+\varepsilon}, \quad m\left(r, \frac{1}{f}\right)<r^{\rho-1+\varepsilon},
$$

and

$$
N(r, f)<r^{\rho-1+\varepsilon}, \quad N\left(r, \frac{1}{f}\right)<r^{\rho-1+\varepsilon} .
$$

It follows from (6), if $\varepsilon$ is an arbitrary positive number then for all values of $r>r_{0}(\varepsilon)$

$$
(2 r)^{\rho-1+\varepsilon}>N(2 r, f) \geq \int_{r}^{2 r} \frac{\lambda_{-}(t)}{t^{3}} d t \geq \lambda_{-}(r) \int_{r}^{2 r} \frac{d t}{t^{3}}=\frac{3 \lambda_{-}(r)}{8 r^{2}} .
$$

From this and similar inequality for $\lambda_{+}(r)$ we obtain asymptotic inequalities

$$
\lambda_{+}(r)<r^{\rho+1+\varepsilon}, \quad \lambda_{-}(r)<r^{\rho+1+\varepsilon} .
$$

Let $m_{2}(r, f)$ be $L_{2}$-norm of the function $f$ on the semicircle of $\left\{r e^{i \theta}: 0 \leq \theta \leq \pi\right\}$ :

$$
m_{2}(r, f)=\left\{\frac{1}{\pi} \int_{0}^{\pi}|\log | f\left(r e^{i \theta}\right)||^{2} d \theta\right\}^{1 / 2} .
$$

The main result is following theorem. 
Theorem 1. Let $f \in J M(\rho), \rho>1$. Then

$$
\limsup _{r \rightarrow \infty} \frac{r(N(r, f)+N(r, 1 / f))}{m_{2}(r, f)} \geq \frac{|\sin \pi \rho|}{\rho^{2}-1} \sqrt{1 /\left(1-\frac{\sin 2 \pi \rho}{2 \pi \rho}\right)},
$$

and this inequality is sharp, i. e. for some meromorphic function $f, f \in J M(\rho), \rho>1$, equality (8) holds.

Corollary. Let $f \in J M(\rho), \rho>1$. Then

$$
\varkappa(f):=\limsup _{r \rightarrow \infty} \frac{N(r, f)+N(r, 1 / f)}{T(r, f)} \geq \frac{5|\sin \pi \rho|}{7\left(\rho^{2}-1\right)} .
$$

1. Fourier coefficients of a function. The Fourier coefficients of a function $f \in J M$ are defined as usual [5]:

$$
c_{k}(r, f)=\frac{2}{\pi} \int_{0}^{\pi} \log \left|f\left(r e^{i \theta}\right)\right| \sin k \theta d \theta, \quad k \in \mathbb{N} .
$$

From (1) we obtain the following expressions for the Fourier coefficients for $r>r_{0}$ :

$$
c_{k}(r, f)=\alpha_{k} r^{k}+\frac{2 r^{k}}{\pi} \int_{r_{0}}^{r} \frac{\lambda_{k}(t)}{t^{2 k+1}} d t, \quad k \in \mathbb{N},
$$

where $\alpha_{k}=r_{0}^{-k} c_{k}\left(r_{0}, v\right)$.

Applying the formula of integration by parts to the integral in (10), we obtain

$$
\begin{gathered}
c_{k}(r, f)=\alpha_{k} r^{k}+\frac{r^{k}}{\pi k r_{0}^{2 k}} \iint_{C_{+}\left(0, r_{0}\right)} \frac{\sin k \varphi}{\operatorname{Im} \zeta} \tau^{k} d \lambda(\zeta)+ \\
+\frac{r^{k}}{\pi k} \iint_{r_{0} \leq|\zeta| \leq r} \frac{\sin k \varphi}{\tau^{k} \operatorname{Im} \zeta} d \lambda(\zeta)-\frac{1}{r^{k} \pi k} \iint \frac{\sin k \varphi}{\operatorname{Im} \zeta} \tau^{k} d \lambda(\zeta), \zeta=\tau e^{i \varphi},
\end{gathered}
$$

where the kernel $\frac{\sin k \varphi}{\tau^{k} \operatorname{Im} \zeta}$ is extended by continuity to the points on the real axis.

The following theorem is a corollary of Theorem 4 from [9].

Theorem 2. Let $f \in J M(\rho)$ be a just meromorphic function of finite order $\rho>1, p=[\rho]$. Let $\lambda$ be the complete measure of the function $f(z), \lambda_{1}$ be the restriction of $\lambda$ to the disc $C(0,1)$, and $\lambda_{2}=\lambda-\lambda_{1}$. Then there exist real numbers $\left\{d_{k}\right\}_{k=1}^{p}$ such that

$$
\log |f(z)|=\frac{1}{2 \pi} \iint K_{0}(z, \zeta) d \lambda_{1}(\zeta)+\frac{1}{2 \pi} \iint K_{p}(z, \zeta) d \lambda_{2}(\zeta)+\sum_{k=1}^{p} d_{k} \operatorname{Im} z^{k}, \quad p=[\rho]
$$

where

$$
K_{p}(z, \zeta)=\frac{1}{\operatorname{Im} \zeta} \operatorname{Re}\left[\log \frac{z-\zeta}{z-\bar{\zeta}}+\sum_{k=1}^{p} \frac{z^{k}}{k}\left(\frac{1}{\zeta^{k}}-\frac{1}{\bar{\zeta}^{k}}\right)\right], \quad p=1,2, \ldots
$$


2. Fourier coefficients of a pair. Let here and below throughout this section $\lambda$ be a non-negative measure in $\overline{\mathbb{C}}_{+}$. For $k \in \mathbb{N}$ and the measure $\lambda$, we denote

$$
\begin{gathered}
S_{+}(r ; k):=S_{+}(r ; k, \lambda)=\frac{1}{\pi k} \iint_{r_{0} \leq|\zeta| \leq r} \frac{\sin k \varphi}{\tau^{k} \operatorname{Im} \zeta} d \lambda(\zeta)+\frac{1}{\pi k r_{0}^{2 k}} \iint \frac{\sin k \varphi}{\operatorname{Im} \zeta} \tau^{k} d \lambda(\zeta), \\
S_{+}\left(r_{1}, r_{2} ; k\right):=S_{+}\left(r_{1}, r_{2} ; k, \lambda\right)=S_{+}\left(r_{2} ; k\right)-S_{+}\left(r_{1} ; k\right), \quad r_{1} \leq r_{2}, \\
S_{+}^{\prime}(r ; k):=S_{+}^{\prime}(r ; k, \lambda)=\frac{1}{\pi k r^{k}} \iint \frac{\tau^{k} \sin k \varphi}{\operatorname{Im} \zeta} d \lambda(\zeta), \quad N(r, \lambda):=\int_{r_{0}}^{r} \frac{\lambda(t)}{t^{3}} d t,
\end{gathered}
$$

where $\zeta=\tau e^{i \varphi}, r_{0}>0$ is a fixed number.

Lemma 2. The following inequality holds:

$$
\left|S_{+}^{\prime}(r ; k)\right| \leq \frac{2 N(2 r, \lambda) r}{3 \pi}
$$

Proof. The proof is provided by the inequalities

$$
\left|S_{+}^{\prime}(r ; k)\right| \leq \frac{\lambda(r)}{\pi r}=\frac{2 \lambda(r) r}{3 \pi} \int_{r}^{2 r} \frac{d(t)}{t^{3}} \leq \frac{2 r}{3 \pi} \int_{r}^{2 r} \frac{\lambda(t)}{t^{3}} d(t) \leq \frac{2 r}{3 \pi} \int_{r_{0}}^{2 r} \frac{\lambda(t)}{t^{3}} d(t)=\frac{2 N(2 r, \lambda) r}{3 \pi} .
$$

Definition 2. A measure $\lambda$ has finite $\rho$-density if for any $\varepsilon>0$ there exists $r_{0}=r_{0}(\varepsilon)$ such that

$$
N(r, \lambda) \leq r^{\rho-1+\varepsilon}
$$

for all $r>r_{0}$.

Definition 3. A measure $\lambda$ has finite order $\rho$ if for any $\varepsilon>0$ there exists $r_{0}=r_{0}(\varepsilon)$ such that

$$
\lambda(r):=\lambda(C(0, r)) \leq r^{\rho+1+\varepsilon}
$$

for all $r>r_{0}$.

Lemma 3. If $\lambda$ is measure of finite $\rho$-density, then it is measure of finite order $\rho$.

Proof. The proof is provided by the inequalities

$$
N(2 r, \lambda)=\int_{r_{0}}^{2 r} \frac{\lambda(t)}{t^{3}} d t \geq \int_{r}^{2 r} \frac{\lambda(t)}{t^{3}} d t \geq \frac{3 \lambda(r)}{3 r^{2}}
$$

Definition 4. A measure $\lambda$ is said to be $\rho$-admissible if for any $\varepsilon>0$ there exists $r_{0}=r_{0}(\varepsilon)$ such that

$$
\left|S_{+}\left(r_{1}, r_{2} ; k, \lambda\right)\right| \leq \frac{r_{1}^{\rho+\varepsilon}}{r_{1}^{k}}+\frac{r_{2}^{\rho+\varepsilon}}{r_{2}^{k}}, \quad k=2,3, \ldots,
$$

for all $r_{2}>r_{1}>r_{0}$ and it has finite $\rho$-density.

The following lemma is true.

Lemma 4. Let measure $\lambda$ have finite $\rho$-density. Then the following properties are equivalent: 
(1) $\lambda$ is $\rho$-admissible;

(2) there exists a sequence of real numbers $\beta=\left\{\beta_{k}\right\}$ such that for all $\varepsilon>0$ and $k \in \mathbb{N}$ we have

$$
\left|\beta_{k}+S_{+}(r ; k, \lambda)\right| \leq \frac{r^{\rho+\varepsilon}}{r^{k}}
$$

for all $r>r_{0}=r_{0}(\varepsilon)$.

Proof. Lemma 4 is a consequence of Theorem 3 from [5].

Properties (14) of the measure $\lambda$ and have finite $\rho$-density are independent. Note that the condition of finite $\rho$-density is equivalent to (14) for $k=1$. In fact, let (14) hold for $k=1$. Then it follows from

$$
\frac{\lambda(r)}{r^{2}}-\frac{\lambda\left(r_{0}\right)}{r_{0}^{2}}+\frac{2}{\pi} \int_{r_{0}}^{r} \frac{\lambda(t)}{t^{3}} d t=\frac{1}{\pi} \int_{r_{0}}^{r} \frac{d \lambda(t)}{t^{2}}=\iint_{r_{0} \leq|\zeta| \leq r} \frac{d \lambda(\zeta)}{\tau^{2}} \leq \frac{r_{0}^{\rho+\varepsilon}}{r_{0}}+\frac{r^{\rho+\varepsilon}}{r}
$$

that $\lambda$ has finite $\rho$-density.

Conversely, let $\lambda$ have finite $\rho$-density and let $\varepsilon>0$ be any number. Using (13) we obtain

$$
\begin{gathered}
\left|S_{+}\left(r_{1}, r_{2} ; 1, \lambda\right)\right| \leq \frac{1}{\pi} \iint_{r_{1} \leq|\zeta| \leq r_{2}} \frac{d \lambda(\zeta)}{\tau^{2}}=\frac{1}{\pi} \int_{r_{1}}^{r_{2}} \frac{d \lambda(t)}{t^{2}}=\frac{\lambda\left(r^{2}\right)}{r_{2}^{2}}-\frac{\lambda\left(r_{1}\right)}{r_{1}^{2}}+\frac{2}{\pi} \int_{r_{1}}^{r_{2}} \frac{\lambda(t)}{t^{3}} d t \leq \\
\leq \frac{r_{2}^{\rho+\varepsilon}}{r_{2}}-\frac{\lambda\left(r_{1}\right)}{r_{1}^{2}}+\frac{2}{\pi} \int_{r_{1}}^{r_{2}} t^{\rho+\varepsilon-2} d t=\frac{\pi(\rho+\varepsilon-2)+2}{\pi(\rho+\varepsilon-1)} \cdot \frac{r_{2}^{\rho+\varepsilon}}{r_{2}}-\frac{\pi(\rho+\varepsilon-2)+2}{\pi(\rho+\varepsilon-1)} \cdot \frac{\lambda\left(r_{1}\right)}{r_{1}^{2}} .
\end{gathered}
$$

Definition 5. Let $\beta=\left\{\beta_{k}\right\}$ be a some sequence of real numbers. The functions

$$
c_{k}(r ; \lambda, \beta)=r^{k}\left\{\beta_{k}+S_{+}(r ; k, \lambda)\right\}-S_{+}^{\prime}(r ; k, \lambda), \quad k \in \mathbb{N},
$$

is called Fourier coefficients of the pair $(\lambda, \beta)$.

Definition 6. A pair $(\lambda, \beta)$ is said to be $\rho$-admissible if for any $\varepsilon>0$ there exists $r_{0}=r_{0}(\varepsilon)$ such that

$$
\left|c_{k}(r ; \lambda, \beta)\right| \leq r^{\rho+\varepsilon}, \quad k \in \mathbb{N},
$$

for all $r>r_{0}=r_{0}$ and the measure $\lambda$ has finite $\rho$-density.

Lemma 5. A measure $\lambda$ is $\rho$-admissible if and only if there exists a sequence $\beta$ such that the pair $(\lambda, \beta)$ is $\rho$-admissible.

Proof. Let $\lambda$ be $\rho$-admissible measure. Then by Lemma 4 property (2) holds and we can take for $\beta$ the sequence in (15). The relation (17) follows for $k \in \mathbb{N}$ from the inequality

$$
\left|c_{k}(r ; \lambda, \beta)\right| \leq r^{k}\left|\beta_{k}+S_{+}(r ; k, \lambda)\right|+\left|S_{+}^{\prime}(r ; k, \lambda)\right|
$$

Lemma 3 and our choice of $\beta$. 
Assume now that (17) holds and the measure $\lambda$ has finite $\rho$-density. Then for $k \in \mathbb{N}$ we have by Lemma 3

$$
r^{k}\left|\beta_{k}+S_{+}(r ; k, \lambda)\right|=\left|c_{k}(r ; \lambda, \beta)+S_{+}^{\prime}(r ; k, \lambda)\right| \leq r^{\rho+\varepsilon}+\frac{2 N(2 r, \lambda) r}{3 \pi} .
$$

Thus, (15) holds. By Lemma $4 \lambda$ is $\rho$-admissible measure.

The following lemma is a consequence of Theorem 1, Theorem 5 and Theorem 6 from [5].

Lemma 6. If a pair $(\lambda, \beta)$ is $\rho$-admissible then there exists only one function $f \in J A(\rho)$ such that $c_{k}(r, f)=c_{k}(r ; \lambda, \beta)$.

3. Proof of Theorem 1. Let us prove Theorem 1. Let $f \in J M(\rho), \rho>1$.

To prove this theorem, we need the following lemma.

Lemma 7. Let $f \in J M(\rho(r)), \lim _{r \rightarrow+\infty} \rho(r)=\rho>1$, and let $\lambda=\lambda_{+}-\lambda_{-}$be the complete measure of $f$. Then for all $k>\rho$

$$
c_{k}(r, f)=-\frac{r^{k}}{\pi k} \iint_{|\zeta| \geq r} \frac{\sin k \varphi}{\tau^{k} \operatorname{Im} \zeta} d \lambda(\zeta)+\frac{1}{r^{k} \pi k} \iint_{\overline{C_{+}(0, r)}} \frac{\sin k \varphi}{\operatorname{Im} \zeta} \tau^{k} d \lambda(\zeta), \zeta=\tau e^{i \varphi} .
$$

Proof. By (11), we have

$$
\begin{gathered}
r^{k}\left|\alpha_{k}+\frac{1}{\pi k r_{0}^{2 k}} \iint_{C_{+}\left(0, r_{0}\right)} \frac{\sin k \varphi}{\operatorname{Im} \zeta} \tau^{k} d \lambda(\zeta)+S_{+}\left(r ; k, \lambda_{+}\right)-S_{+}\left(r ; k, \lambda_{-}\right)\right| \leq \\
\leq\left|c_{k}(r, f)\right|+\left|S_{+}^{\prime}\left(r ; k, \lambda_{+}-S_{+}^{\prime}\left(r ; k, \lambda_{-}\right)\right)\right|,
\end{gathered}
$$

We divide the inequality (19) by $r^{k}, k>\rho$, and pass to the limit as $r \rightarrow \infty$. Using the inequalities $\left|c_{k}(r, f)\right| \leq 2 T(r, f)$ and

$$
\left|S_{+}^{\prime}\left(r ; k, \lambda_{+}-S_{+}^{\prime}\left(r ; k, \lambda_{-}\right)\right)\right| \leq \frac{2 r}{3 \pi}\left(N\left(r, \lambda_{+}\right)+N\left(r, \lambda_{-}\right)\right)
$$

we obtain that the right-part of (19) tends to zero. Then we obtain

$$
\alpha_{k}+\frac{1}{\pi k r_{0}^{2 k}} \iint_{C_{+}\left(0, r_{0}\right)} \frac{\sin k \varphi}{\operatorname{Im} \zeta} \tau^{k} d \lambda(\zeta)=-\left(S_{+}\left(+\infty ; k, \lambda_{+}\right)-S_{+}\left(+\infty ; k, \lambda_{-}\right)\right), \quad k>\rho .
$$

Substituting this value in (11), we obtain (18).

Let $\rho$ be non-integer. In the case of integer $\rho$, the theorem is obvious.

We define the measure $\tilde{\lambda}$ by the equality $\tilde{\lambda}=\left|\lambda_{f}\right|$. Without loss of generality, we can suppose that the measure $\lambda_{f}$ does not load some neighborhood of zero. Denote by $q=[\rho]$, $\beta=\left\{\beta_{k}\right\}$

$$
\beta_{k}= \begin{cases}\left|\alpha_{k}(f)\right|+\left|\frac{1}{\pi k r_{0}^{2 k}} \iint_{C_{+}\left(0, r_{0}\right)} \frac{\sin k \varphi}{\operatorname{Im} \zeta} \tau^{k} d \lambda_{f}(\zeta)\right|, & 1<k<q+1 \\ -S_{+}(+\infty ; k, \tilde{\lambda}), & k \geq q+1,\end{cases}
$$


where

$$
S_{+}(+\infty ; k, \tilde{\lambda}):=\lim _{r \rightarrow \infty} S_{+}(r ; k, \tilde{\lambda}) .
$$

It is clear that $\tilde{\lambda}(r)=\left|\lambda_{f}\right|(r)$. For some $A>0, \varepsilon>0, \rho+\varepsilon<q+1$, we have $\tilde{\lambda}(r) \leq A r^{\rho+1+\varepsilon}$, $r>r_{0}$. Then for $k \geq q+1$ we obtain

$$
c_{k}(r ; \tilde{\lambda}, \beta)=-\frac{r^{k}}{\pi k} \iint_{|\zeta| \geq r} \frac{\sin k \varphi}{\tau^{k} \operatorname{Im} \zeta} d \tilde{\lambda}(\zeta)-\frac{1}{\pi k r^{k}} \iint_{\overline{C_{+}(0, r)}} \frac{\tau^{k} \sin k \varphi}{\operatorname{Im} \zeta} d \tilde{\lambda}(\zeta) .
$$

From this

$$
\left|c_{k}(r ; \tilde{\lambda}, \beta)\right| \leq \frac{1}{r^{k} \pi} \int_{0}^{r} t^{k-1} d \tilde{\lambda}(t)+\frac{r^{k}}{\pi} \int_{r}^{\infty} \frac{d \tilde{\lambda}(t)}{t^{k+1}}, \quad k \geq q+1 .
$$

By integrating in parts, we obtain the estimate

$$
\begin{aligned}
& \left|c_{k}(r ; \tilde{\lambda}, \beta)\right| \leq \frac{(k+1) r^{k}}{\pi} \int_{r}^{\infty} \frac{\tilde{\lambda}(t)}{t^{k+2}} d t-\frac{k-1}{r^{k} \pi} \int_{0}^{r} t^{k-2} \tilde{\lambda}(t) d t \leq \\
& \leq \frac{A(k+1) r^{k}}{\pi} \int_{r}^{\infty} t^{\rho+\varepsilon-k-1} d t=\frac{A(k+1) r^{\rho+\varepsilon}}{\pi(k-\rho-\varepsilon)}, \quad k \geq q+1 .
\end{aligned}
$$

That is

$$
\left|c_{k}(r ; \tilde{\lambda}, \beta)\right| \leq \frac{A(k+1) r^{\rho+\varepsilon}}{\pi(k-\rho-\varepsilon)}, \quad k \geq q+1
$$

Obviously, epy similar inequality holds for $1 \leq k<q+1$. Thus the pair $(\tilde{\lambda}, \beta)$ is $\rho$ admissible. By Lemma 6 , there exists the function $\bar{F} \in J A(\rho)$ such that $c_{k}(r, F)=c_{k}(r ; \tilde{\lambda}, \beta)$ for all $r>0$ and for all $k \in \mathbb{N}$.

Set

$$
N_{1}(r)=N_{1}(r, F):=\int_{1}^{r} \frac{\tilde{\lambda}(t)}{t^{3}} d t
$$

The order of the function $r N(r)$ is less than or equal to $\rho$. In fact, it is equal $\rho$, because otherwise it follows from Theorem $2.3[9]$ that this order of $T(r, f)$ is integer, but we excluded this case.

By integrating by parts in (20), we obtain

$$
\begin{gathered}
\left|c_{k}(r, F)\right| \leq \frac{(k+1) r^{k}}{\pi} \int_{0}^{r} \frac{d N_{1}(t)}{t^{k-1}}-\frac{k-1}{r^{k} \pi} \int_{0}^{r} t^{k+1} d N_{1}(t)= \\
=\frac{k^{2}-1}{\pi}\left\{\int_{0}^{r}\left(\frac{t}{r}\right)^{k} N_{1}(t) d t+\int_{r}^{\infty}\left(\frac{r}{t}\right)^{k} N_{1}(t) d t\right\}-\frac{2 k}{\pi} r N_{1}(r), \quad k \geq q+1 .
\end{gathered}
$$

And for $1 \leq k \leq q$, we have

$$
\left|c_{k}(r, F)\right| \leq r^{k}\left(\left|\alpha_{k}\right|+\frac{2}{\pi r_{0}^{2 k}} \int_{0}^{r_{0}} t^{k-1} d \tilde{\lambda}(t)\right)+\frac{1}{\pi} \int_{r_{0}}^{r}\left[\left(\frac{r}{t}\right)^{k}-\left(\frac{t}{r}\right)^{k}\right] \frac{d \tilde{\lambda}(t)}{t}, \quad 1 \leq k \leq q .
$$


From this, by double integration by parts, we obtain the inequality

$$
\left|c_{k}(r, F)\right| \leq r^{k} \gamma_{k}+\frac{2 k}{\pi} r N_{1}(r)+\frac{k^{2}-1}{\pi} \int_{r_{0}}^{r}\left[\left(\frac{r}{t}\right)^{k}-\left(\frac{t}{r}\right)^{k}\right] N_{1}(t) d t, 1 \leq k \leq q,
$$

where

$$
\gamma_{k}=\left|\alpha_{k}\right|+\frac{2}{\pi r_{0}^{2 k}} \int_{0}^{r_{0}} t^{k-1} d \tilde{\lambda}(t)+\frac{\tilde{\lambda}\left(r_{0}\right)}{\pi r_{0}^{k+1}} .
$$

Besides, $\left|c_{k}(r, f)\right| \leq\left|c_{k}(r, F)\right|, 1 \leq k \leq q$, and $\left|c_{k}(r, f)\right| \leq-c_{k}(r, F), k \geq q+1$. From this

$$
m_{2}(r, f) \leq m_{2}(r, F)
$$

Let $\varepsilon>0$ be a fixed number. Applying the lemma of Pólya peaks [10] for functions $r N_{1}(r), r^{\rho-\varepsilon}, r^{\rho+\varepsilon}$, we find the sequence $\left(r_{n}\right), \lim _{n \rightarrow \infty} r_{n}=\infty,[7$, p. 62] such that

$$
t N_{1}(t) \leq\left(\frac{t}{r_{n}}\right)^{\rho-\varepsilon} r_{n} N_{1}\left(r_{n}\right), 0<t \leq r_{n}, \quad t N_{1}(t) \leq\left(\frac{t}{r_{n}}\right)^{\rho+\varepsilon} r_{n} N_{1}\left(r_{n}\right), t>r_{n} .
$$

Using inequalities (21), (22), (24), we obtain

$$
\begin{array}{cl}
\left|c_{k}\left(r_{n}, f\right)\right| \leq r_{n}^{k} \gamma_{k}+\frac{2 k}{\pi} r_{n} N_{1}\left(r_{n}\right)\left(\frac{k^{2}-1}{(\rho-\varepsilon)^{2}-k^{2}}+1\right), & 1 \leq k \leq[\rho], \\
\left|c_{k}\left(r_{n}, f\right)\right| \leq \frac{2}{\pi} r_{n} N_{1}\left(r_{n}\right)\left(\frac{\left(k^{2}-1\right)(k-\varepsilon)}{(\rho-\varepsilon)^{2}-k^{2}}-k\right), & k>[\rho] .
\end{array}
$$

Inequality (24) implies, in particular, that $r_{n}^{[\rho]}=o\left(r_{n} N_{1}\left(r_{n}\right)\right)$ as $n \rightarrow \infty$, since for $t^{\prime}$ such that $r_{0}<t^{\prime}$ and $t^{\prime} N_{1}\left(t^{\prime}\right) \geq\left(t^{\prime}\right)^{\rho-\varepsilon}$ for all $r_{n} \geq t^{\prime}$ the inequality

$$
r_{n}^{q-\varepsilon}<r_{n}^{\rho-\varepsilon} \leq r_{n}^{\rho-\varepsilon} \frac{t^{\prime} N_{1}\left(t^{\prime}\right)}{\left(t^{\prime}\right)^{\rho-\varepsilon}} \leq r_{n} N_{1}\left(r_{n}\right)
$$

holds if $2 \varepsilon<\rho-q$.

From this remark, arbitrariness $\varepsilon$ and (25), it follows that

$$
\limsup _{n \rightarrow \infty} \frac{\left|c_{k}\left(r_{n}, f\right)\right|}{r_{n} N_{1}\left(r_{n}\right)} \leq \frac{2 k}{\pi} \frac{\rho^{2}-1}{\left|\rho^{2}-k^{2}\right|}, \quad k=1,2, \ldots
$$

By the Parseval equality, we obtain the inequality

$$
\liminf _{n \rightarrow \infty} \frac{m_{2}(r, F)}{r N_{1}(r)} \leq\left\{\sum_{k=-\infty}^{\infty} \frac{2 k^{2}}{\pi^{2}} \frac{\left(\rho^{2}-1\right)^{2}}{\left|\rho^{2}-k^{2}\right|^{2}}\right\}^{1 / 2} .
$$

The sum on the right-hand side of this inequality can be easily found using residues [11]. It is equal to

$$
\sum_{k=-\infty}^{\infty} \frac{2 k^{2}}{\pi^{2}} \frac{\left(\rho^{2}-1\right)^{2}}{\left|\rho^{2}-k^{2}\right|^{2}}=\left(\frac{\rho^{2}-1}{|\sin \pi \rho|}\right)^{2}\left(1-\frac{\sin 2 \pi \rho}{2 \pi \rho}\right)
$$


Since $N_{1}(r)=N(r, f)+N(r, 1 / f)$, by (26) we get the statement of Theorem 1 .

We show that the estimate $(8)$ is sharp.

Example. By Theorem 2 the function

$$
f(z)=\exp \left\{z^{q+1} \int_{|t| \geq r_{0}} \frac{t^{\rho} d t}{(t-z) t^{q+1}}\right\}=\exp \left\{z^{q+1} \int_{|t| \geq r_{0}} \frac{t^{\rho-q-1} d t}{t-z}\right\}, \quad 1<\rho \notin \mathbb{N}, q=[\rho],
$$

is an analytic function in $\mathbb{C}_{+}$of order $\rho>1$ with the complete measure $\lambda_{f}(t)=t^{\rho+1}=$ $\lambda_{+}(t)=\tilde{\lambda}(t)$ if $t \in \mathbb{R},|t| \geq r_{0}$, and $d \lambda_{f}(z) \equiv 0$ if $z \in \mathbb{C}_{+} \cup\left(-r_{0}, r_{0}\right)$,

$$
N_{1}(r)=N(r, 1 / f)=\frac{r^{\rho-1}}{\rho-1}-\frac{1}{\rho-1}, N(r, f) \equiv 0, \lambda_{k}(t)=\frac{k(\rho+1)}{k+\rho} t^{k+\rho} .
$$

By (20), we obtain

$$
c_{k}(r, f)=-\frac{2 r^{k} k(\rho+1)}{\pi(k+\rho)} \int_{r}^{\infty} t^{\rho-k-1} d t=\frac{2 r^{\rho} k(\rho+1)}{\pi\left(k^{2}-\rho^{2}\right)}, \quad k>[\rho] .
$$

Then

$$
\frac{c_{k}(r, f)}{r N(r, 1 / f)}=\frac{2 r^{\rho} k\left(\rho^{2}-1\right)}{\pi\left(k^{2}-\rho^{2}\right)}+o(1), \quad r \rightarrow \infty, k>[\rho] .
$$

By (11), we obtain further

$$
\begin{aligned}
& c_{k}(r, f)=r^{k} \tilde{\gamma}+\frac{\rho+1}{\pi} \int_{r_{0}}^{r}\left[\left(\frac{r}{t}\right)^{k}-\left(\frac{t}{r}\right)^{k}\right] t^{\rho-1} d t= \\
& =r^{k} \tilde{\gamma}+\frac{2 r^{\rho} k\left(\rho^{2}-1\right)}{\pi\left(k^{2}-\rho^{2}\right)}+o(1), \quad r \rightarrow \infty, 1 \leq k \leq[\rho],
\end{aligned}
$$

where $\tilde{\gamma}=\alpha_{k}+\frac{2}{\pi r_{0}^{2 k}} \int_{0}^{r_{0}} t^{k-1} d \tilde{\lambda}(t)$.

From this and (27), we obtain

$$
\lim _{r \rightarrow \infty} \frac{c_{k}(r, f)}{r N(r, 1 / f)}=\frac{2 k\left(\rho^{2}-1\right)}{\pi\left(k^{2}-\rho^{2}\right)}, \quad k \in \mathbb{N} .
$$

Thus, for $f(z)$ the estimate (8) is sharp.

Theorem 1 is proved.

3. Analogue of the Nevanlinna problem for half-plane. Let us prove the corollary related to the analogue of the Nevanlinna problem for half-plane. By the Carleman formula (2), we have

$$
\begin{gathered}
m_{2}(r, f) \geq m_{1}(r, f) \geq r[m(r, f)+m(r, 1 / f)]= \\
=2 r T(r, f)-r\left[N(r, f)+N(r, 1 / f)+m\left(r_{0}, f\right)+m\left(r_{0}, 1 / f\right)\right] .
\end{gathered}
$$

By (8) and inequality

$$
\sqrt{1-\frac{\sin 2 \pi \rho}{2 \pi \rho}} \leq 1+\frac{1}{4 \pi \rho}
$$


we obtain

$$
\varkappa(f) \geq \frac{|\sin \pi \rho|}{\rho^{2}-1} /\left(\frac{1}{2}+\frac{1}{8 \pi \rho}+\frac{|\sin \pi \rho|}{2\left(\rho^{2}-1\right)}\right) .
$$

Since

$$
\frac{1}{2}+\frac{1}{8 \pi \rho}+\frac{|\sin \pi \rho|}{2\left(\rho^{2}-1\right)} \leq \frac{2 \pi^{2}+4 \pi+1}{8 \pi},
$$

we obtain the inequality

$$
\varkappa(f) \geq \frac{8 \pi}{2 \pi^{2}+4 \pi+1} \frac{|\sin \pi \rho|}{\rho^{2}-1} .
$$

In conclusion, we note the inequality

$$
\frac{2 \pi^{2}+4 \pi+1}{8 \pi}<\frac{7}{5}
$$

which implies (9).

Acknowledgment. The authors are thankful to the referee for valuable suggestions towards the improvement of the paper.

The reported study was funded by RFBR according to the research project No 18-01-00236.

\section{REFERENCES}

1. L.A. Rubel, B.A. Taylor, A Fourier series method for meromorphic and entire function, Bull. Soc. Math. France, 96 (1968), 53-96.

2. A.A. Kondratyuk, The Fourier series method for entire and meromorphic functions of completely regular growth. I, Mat. Sb. 106 (1978), №148, 386-408; English transl. in Math. USSR-Sb., 35 (1979).

3. A.A. Kondratyuk, The Fourier series method for entire and meromorphic functions of completely regular growth. II, Mat. Sb. 113 (1980), №155, 118-132; English transl. in Math. USSR-Sb., 41 (1982).

4. A.A. Kondratyuk, The Fourier series method for entire and meromorphic functions of completely regular growth. III, Mat. Sb. 120 (1983), №162, 331-343; English transl. in Math. USSR-Sb., 48 (1984).

5. K.G. Malyutin, Fourier series and $\delta$-subharmonic functions of finite $\gamma$-type in a half-plane, Sbornik: Mathematics, 192 (2001), №6, 843-861. doi : 10.1070/SM2001v192n06ABEH000572

6. J.B. Miles, D.P. Shea, An extremal problem in value distribution theory, Quart. J. Math. Oxford, 24 (1973), 377-383.

7. A.A. Kondratyuk, Fourier Series and Meromorphic Functions, Vyshcha shkola, Lviv, 1988. (in Russian)

8. M.A. Fedorov, A.F. Grishin, Some Questions of the Nevanlinna Theory for the Complex Half-Plane, Mathematical Physics, Analysis and Geometry, 1 (1998), №3, 223-271.

9. K.G. Malyutin, N. Sadik, Representation of subharmonic functions in a half-plane, Sbornik: Mathematics, 198 (2007), №12, 1747-1761. doi : 10.1070/SM2007v198n12ABEH00390

10. Pólya G., Untersuchungen über Lücen and Singularitätin von Potenzreihen, Math. Zeitschrift, 29 (1929), 549-640.

11. J. Bak, D. J. Newman, Complex Analysis, Springer, 2010.

Kursk State University

Department of Mathematical Analysis

Kursk, Russia

malyutinkg@gmail.com

revenko253@mail.ru 